\title{
Teaching Multidisciplinary Engineering Principles Through Environmental Topics
}

\author{
George Piper, Jennifer Waters, Terrence Dwan, Kiriakos Kiriakidis \\ United States Naval Academy
}

\subsection{Introduction}

Environmental topics such as pollution prevention, pollution remediation, natural resource utilization, as well as global and local weather studies provide excellent catalysts to teach a wide breadth of engineering principles. Since environmental issues are real and tangible to most students, engineering applications involving the environment tend to capture the students' interest. Environmental topics also provide a great opportunity for discovery, learning, and meaningful application of learned mathematic, scientific and engineering and principles. For instance, having students explore ways to extract energy from natural resources exposes them to a variety of topics such as thermodynamics, power generation, energy transmission and storage. Through such topics as thermal pollution and weather monitoring, students are exposed to heat transfer, partial differential equations, sensor design, and signal processing. In virtually all environmental topics, students can experience the synthesis of concepts and methods of many different disciplines, which is truly the essence and purpose of engineering.

For the past three years, the Systems Engineering and the Ocean Engineering Departments at the U.S. Naval Academy have collaborated to offer an innovative course sequence in environmental engineering [1]. In this course sequence, a host of engineering principles are explored under the umbrella of environmental topics for non-environmental engineering students. The course sequence is a technical elective track that is offered to systems engineering majors during their senior year. The systems engineering curriculum at the U.S. Naval Academy is a four year, undergraduate, ABET accredited, program specializing in the interaction between mechanical, electrical, and computer systems. The curriculum focuses mainly on linear systems theory, feedback control, and mechatronics. Throughout the curriculum students learn how to model, analyze, and design various types of systems. Prior to the environmental engineering track, the systems engineering students have no formal exposure to environmental engineering. Therefore, environmental engineering track is intended to teach and/or reinforce engineering principles, and then apply them to a real system or problem through environmental topics.

The first course in the track is taught within the Systems Engineering Department. This course reinforces engineering principles taught in the Systems Engineering major [2]. The course concentrates on environmental hardware, sensors, data handling, and modeling. For the second course in the track, students choose from two more traditional environmental courses offered in the Ocean Engineering Department. One course centers on marine pollution, the other course centers on ocean resources. In the following sections each course in this environmental engineering track is discussed, emphasizing their multidisciplinary aspects. 


\subsection{Systems Engineering}

The course taught out of the Systems Engineering Department is entitled Environmental Systems Engineering. It is a survey course covering a wide range of systems engineering topics related to the environment. The course taps into the great diversity of interest and expertise amongst the faculty in the Systems Engineering Department. The course is team-taught typically by an electrical engineer, a mechanical engineer, and a computer scientist. The teamteaching format helps guarantee multidisciplinary instruction better than any other approach. Also, there are usually several environmental engineering professionals who address the class from time to time. Although there is a wide range of topics discussed in the course, the following topics highlight the diversity of the environmental issues and engineering principles taught.

\subsection{Environmental Monitoring, Data Analysis And Modeling}

The monitoring, simulation and analysis of environmental data is accomplished in this course through several weeks of study, laboratory exercises and homework assignments. Each phase of the process will be discussed in depth.

\subsubsection{Measurement of Environmental Data}

Remote sensing has always been an integral part of the environmental engineer's job. In this part of the course each team of two students is given a sensor (temperature, humidity, pressure, etc.) to examine some parameter of the atmospheric environment. The sensors typically produce a very low voltage output proportional to the parameter being measured. There is often a bias voltage present in the signal as well. The teams are responsible for sensor signal processing, calibration, amplification and some circuit hardware. Each team's sensor and signal conditioning circuit is then mounted in our environmental box. The outputs from the sensors are each assigned a channel for data gathering.

The sampling interval for each parameter being measured is set by software (an in-house developed C-program). The final result of sampling the many sensors is a large data file in MATLAB [3] containing time data for each of the sensors. The data is gathered over several weeks.

\subsubsection{Data Analysis of Environmental Data}

Environmental data for the atmosphere will usually contain several components:

- A trend component (seasonal warming, cooling, etc.)

- Two frequency components (daily and seasonal)

- Noise, usually introduced during the measurement process

To familiarize the students with the type of data they will be required to analyze, a simulated signal developed in MATLAB is generated. An example of such a signal is shown in Figure 1. Using Fast Fourier Transforms (FFT) the spectral components may be identified and are shown in Figure 2. Two spectral components are dominant in the simulated data, $50 \mathrm{~Hz}$ and $80 \mathrm{~Hz}$. 


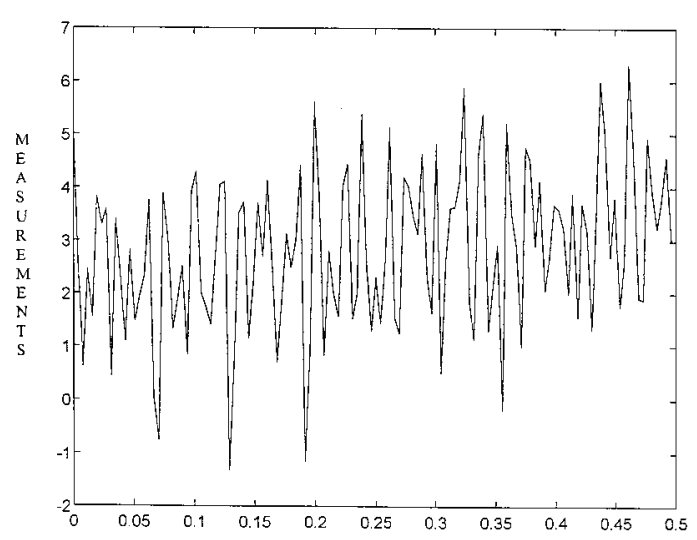

TIME (SEC)

Figure 1.

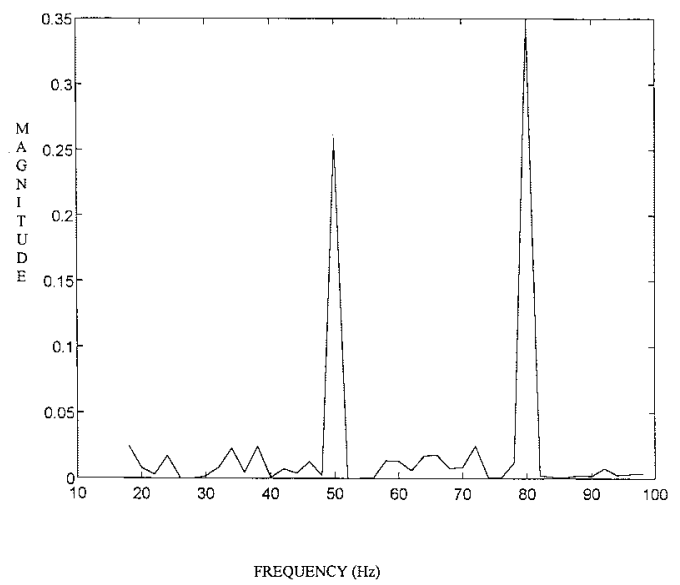

Figure 2.

Once these spectral components have been removed from the data what should remain is the noise (see Figure 3). The data in the simulation is created by adding Gaussian noise, mean of zero and variance of 1.0. It is instructive for the students to examine the statistics of the noise as well as a histogram of the noise is shown in Figure 4.

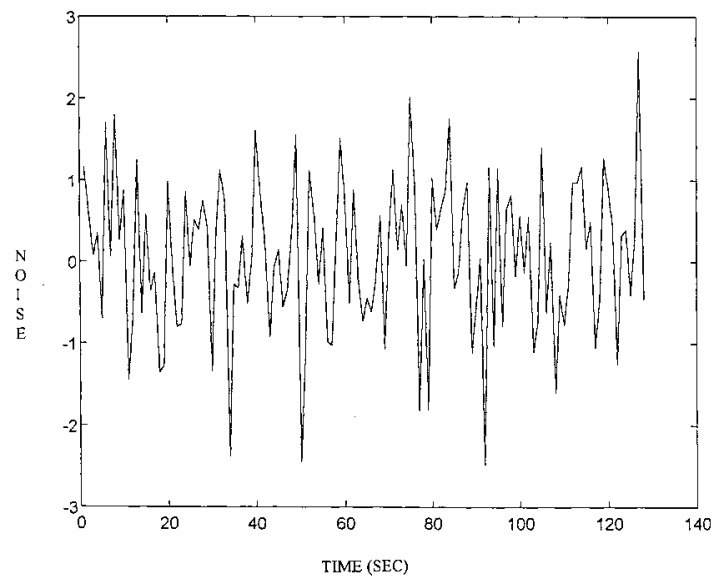

Figure 3.

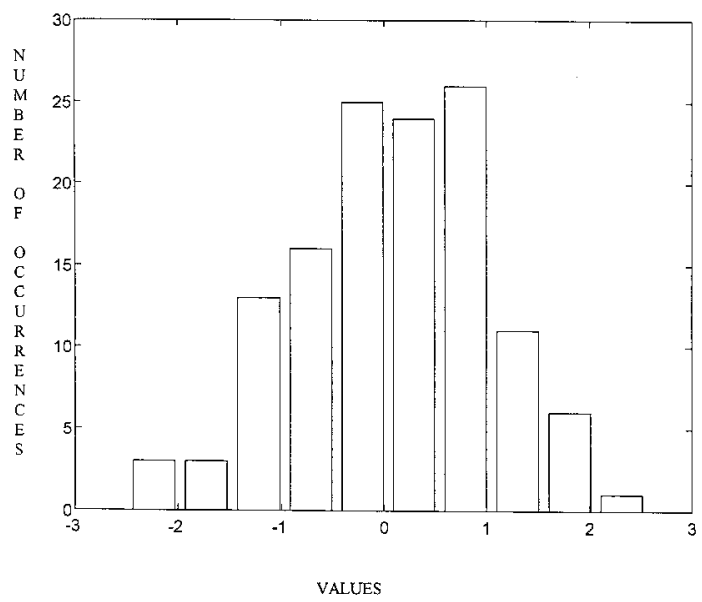

Figure 4.

\subsubsection{Environmental Models}

Each group is required to extract an environmental model from the data gathered by their sensor. The techniques discussed in Section 2.2 are used to find the "best" model for their data. Also, the concepts of filtering, windowing and framing are introduced as the students to facilitate their modeling work. Good, working models are achieved in many instances.

Figure 5 gives the student model derived from the data plotted with the raw data taken for a calcium sulfide photo cell. Figure 6 gives the same results for a pressure sensor. 


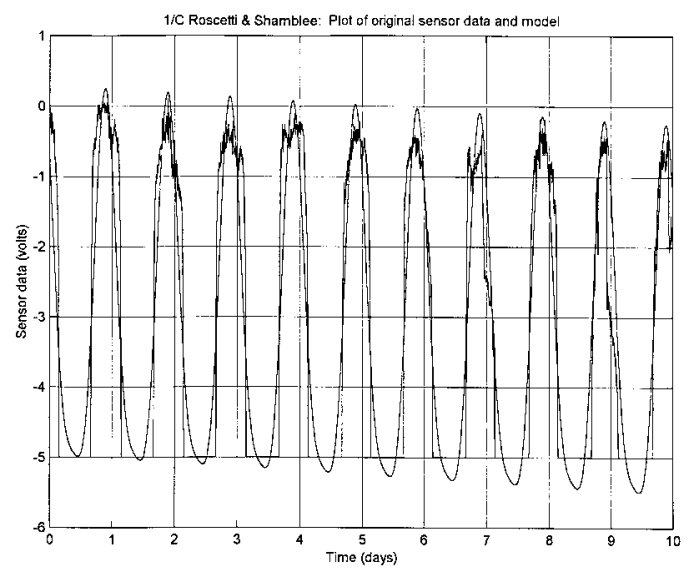

Figure 5.

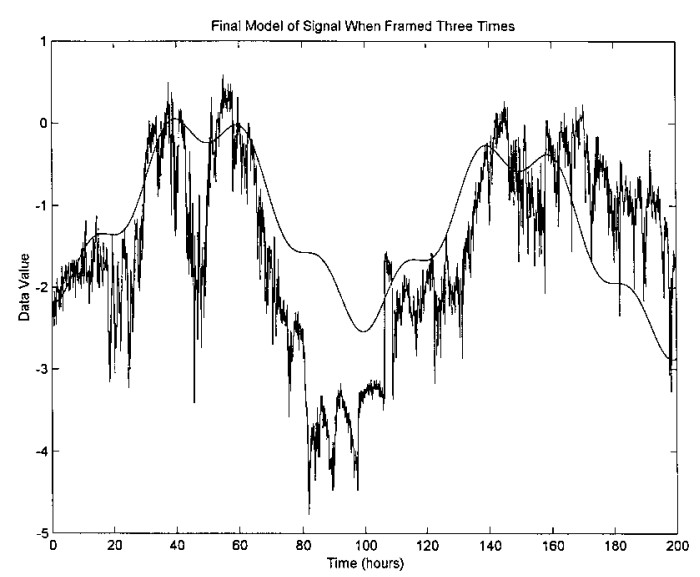

Figure 6.

The students are very pleased with this long sequence: sensor circuit design, data gathering and, finally, in deriving a working model for the environmental parameter under investigation.

\subsection{Alternate Energy Sources}

With the limited supply of oil and coal in the world today, other sources of electricity and power must be developed. Two such sources in use today are hydrodynamic power and solar power. Each source is highly renewable and relatively inexpensive to operate which make them excellent alternative energy sources. A solar panel pumping station is designed by the students to familiarize them with some of the resources available for a viable energy alternative.

In the laboratory students investigate how solar and hydrodynamic power can be used as alternative energy sources. For solar power to be an effective alternative energy source, solar arrays are designed to meet the energy consumption requirements. The correct size, number, and configuration of the solar array are all factors that contribute to an effective solar array. To provide the maximum amount of power during the day the array must be able to track the sun (light source). But what about at night when the sun is no longer able to produce power? For a solar energy source to work around the clock, a storage system must be developed to store power. In the lab, energy storage is accomplished by building up a head of water which can then be tapped to drive a water wheel to provide electricity during the night.

Each team of students designs an array capable of driving a water pump. They also design a light tracking system for the array. The tracking system uses small solar panels as its sensors. The final design must be able to track a light source and pump water from a lower reservoir to a higher reservoir. The stored water offers a source of potential energy which is then taped upon call to run a water wheel. The wheel can then rotate a coil winding to reconvert the stored energy to a more useful electrical form

Another interesting project has the students designing an energy package involving solar arrays and storage batteries to be used on a camping trip. Various specifications are made by the erstwhile camper (boom boxes, TV, etc.). The students are tasked to design a transportable, autonomous system to meet all the specifications. Weight, size and cost are all highly prized 
engineering considerations in their design. Competition for the "best" design is a great learning environment for the students involved.

\subsection{Control of Combustion}

The problem of active combustion control is pivotal in many engineering applications and its impact on the environment conspicuous. At the core of the combustion process, the student finds chemical reactions, which describe the conversion of the air and fuel mixture to a multitude of chemical agents that are harmful to the environment. Only a few of the combustion products, however, are necessary for the complete reaction of the carbon-based fuel with oxygen hence the efficient release of energy. Here, the student learns that tight regulation of the air-to-fuel ratio yields both high efficiency and reduced emissions. To accomplish this, the student will need to apply the methods of the entirely different discipline of automatic control. By demonstrating a nonlinear dynamic behavior, which is difficult to model exactly, the combustion process poses a challenging control problem. After solving such problem and simulating the proposed solution, the student proceeds to the implementation stage. At this point, the student uses knowledge of computer engineering and creates a code that instructs the microprocessor to read from sensors that measure concentration of chemical substances in the flue gases (e.g., excess oxygen), decide the level of correction to inject in the system, and adjust accordingly the actuators (e.g., air-supply solenoid valve).

In conclusion, this exercise brings together the disciplines of chemistry, automatic control, and computer engineering. By balancing theory and practice, it clearly shows the design cycle that is common to all areas of engineering. As with any environmental engineering issue, the goals of combustion control are easy for the student to understand and, therefore, he is able to work on higher level educational objectives such as analysis, synthesis, or evaluation. As an added advantage, the complexity of the exercise necessitates student participation in groups of three hence inducing cooperative learning. The laboratory part of the exercise is very lively. Indeed, the color of the flame provides visual information whether the combustion process is lean or rich and, at the same time, additional stimulation for the student.

\subsection{Mathematical Modeling of Thermal Pollution in Rivers}

Recent years have seen an increased awareness of the detrimental effects of thermal pollution in seas, lakes, and rivers. This awareness has brought a need for a reliable method of predicting the influence of large heat inputs into natural bodies of water. Laboratory modeling of these flows is both difficult and costly. Engineers and scientist have resorted to numerical methods and computer simulations to obtain useful solutions.

To examine how thermal pollutants propagate through the environment, the students are introduced to the concepts of mass and energy transport. In this section, we present a lab where the students learn how to model and simulate the propagation of thermal pollution in a river. The objective of this lab is to use numerical techniques to model the temperature distribution in a river as hot water is discharged upstream.

The lab consist of two parts. In the first part the students measure and record the temperature distribution of an open channel laboratory model as hot water is discharged upstream. Then in the second part of the lab the students mathematically model the temperature distribution with a partial differential equation and solve it using finite difference methods. Finally, the students 
compare the mathematical model to the laboratory model and discuss any differences and similarities.

\subsection{Ocean Engineering}

Systems engineering majors who take the environmental engineering track must choose from two courses offered in the Ocean Engineering Department. Ocean engineering, being one of the most varied engineering disciplines, exposes the systems engineering majors to traditional environment topics and issues.

The two courses offered by the Ocean Engineering Department are titled Ocean Environmental Engineering I and II. These courses are also taken by ocean engineering majors in their environmental engineering track. Each of the two course addresses different aspects of environmental engineering and is independent of the other. Through lectures and laboratory assignments the students are introduced to the basic principles and current issues in environmental engineering as applied to the ocean environment. Each course also incorporates guest speakers and tours of environmental engineering facilities in order to expose students first-hand to a wide variety of environmental engineering design and management issues. Collaborative research projects within each course also allow students to explore more comprehensively certain environmental engineering topics that interest them.

\subsection{Ocean Environmental Engineering I}

The goals of the course are to provide the student with a basic understanding of marine pollution - its principal causes, effects and means of remediation. Students also gain familiarity with environmental laws, ethics and economics as they pertain to the marine environment. Topical coverage includes chemical and biological considerations in water quality; wastewater treatment and discharge; diffusion and dispersion in estuaries and oceanic environments; maintenance dredging and material disposal; engineering methods used to analyze and mitigate the effects of marine pollution; and environmental ethics, economics and regulatory statutes.

The course begins with an introduction to traditional environmental physics and chemistry, since most water pollution issues require an understanding of pollutant types, concentrations, and dispersion characteristics. Key environmental legislation and regulations are then introduced for the first time, although these topics are revisited many times throughout the course. The course then applies these principles and issues to water quality - both groundwater and surface water sources. Groundwater contamination and effects and treatment technologies are discussed, as well as surface water issues such as cultural eutrophication and urban runoff. Conventional water treatment technologies and processes are introduced, which then leads into wastewater treatment technology and plant design issues. For these topics, tours of local water and wastewater treatment plants help to greatly enhance students' understanding of the material.

During the first half of the semester, the students are tasked with selecting a recent ocean environmental engineering journal paper, summarizing it, and presenting a brief talk to the class about the paper, its findings and the student's opinions on it. The student then must develop a web page on the topic, which is published on the course's web site. This exercise helps to motivate and focus the students on current environmental issues, areas of research and development. While the verbal presentation gives students practice in a traditional method of 
fast-paced information transfer, the creation of the web page gives students the opportunity to learn a relatively new mode of publishing and sharing technical information with others.

Toward the end of the semester, engineering aspects of particular current events are highlighted in case studies. Such cases have included oil pollution, containment and cleanup studies, thermal pollution and power plants, as well as other forms of pollutants such as acid rain, pesticides, and radioactivity. The U.S. Navy's pollution prevention afloat (P2A) and base remediation efforts are also discussed within the course. Environmental ethics, economics and risk management are addressed throughout these cases.

In addition to these case studies, students work in teams on an environmental research project of their choice. This team research project addresses a technology or environmental problem related to the course's emphasis. Each research project results in a report, a verbal presentation and another web page placed on the course's web site. Previous research topics have spanned numerous and varied topic areas, including bioremediation, dredging technologies, coral reefs/reef restoration and oil spill cleanup technology. Students generally learn a great deal about a topic that interests them, and frequently comment that they were able to better appreciate many of the principles and issues covered within the course through their research project.

\subsection{Ocean Environmental Engineering II}

This course provides the student with a basic understanding of the environmental, economic and technological aspects associated with recovery of natural resources from the seas. Students also gain familiarity with engineering management technologies for designing effective resource utilization strategies. Issues of environmental law and ethics concerning use of these resources are discussed. Basic principles and current issues in environmental engineering as applied to the ocean environment are introduced. Topical coverage includes the technological aspects of alternate energy sources; deep-ocean oil and gas recovery; desalinization; dredging and uses for dredge spoil; mineral exploitation; ocean depositories; wetlands, reefs and other coastal developments; and environmental economics, ethics and regulatory statutes.

The course has two fundamental emphases: Ocean Energy Recovery and Alternative Ocean Resources. Specific topics covered within the Ocean Energy Recovery portion of the course include

- Ocean Current Energy Conversion,

- Ocean Thermal Energy Conversion,

- Offshore Wind Energy Conversion,

- Tidal Energy/Power Conversion,

- Wave Energy/Power Conversion and

- Alternative Ocean Energy Resources.

Specific topics covered within the alternative ocean resources include

- Artificial Reefs: Function \& Design,

- Aquaculture Technology \& Economics,

- Coral Reefs: Functions and Restoration,

- Dredging Technology and Material Uses,

- Ocean Mineral Recovery (including desalinization), 
- Offshore Oil \& Gas Production,

- Transportation \& Recreation and

- Wetlands: Functions \& Design.

In this course as in the other ocean environmental engineering course, students choose and take on team research projects to enhance the classroom lectures and go into further depth on a particular topic of interest. Through each group's verbal presentation of their findings to the rest of the class, others within the class also gain insight into the other topics.

\subsection{Conclusion}

The systems and ocean engineering courses in the environmental engineering track compliment each other well. These courses expose non-environmental engineering students to a wide variety of environmental topics while reinforcing multidisciplinary engineering principles. The ocean environmental engineering courses provide a sound background in environmental engineering. The systems engineering course, on the other hand, applies system engineering skills to environmental engineering problems. In both courses, the lure of environmental issues provide an excellent catalysts to teach a wide breadth of engineering principles.

The last three offerings of this environmental engineering track for systems engineering majors have been a complete success as evidenced by instructors' evaluations and students' course critiques. Students have demonstrated a good level of mastery of the course material and have received significant exposure to a diverse spectrum of engineering principles. The students have enjoyed the hands-on labs, their research projects, and giving their presentations. The student's enthusiasm is evident by the steadily growing enrollment of the environmental engineering track each year.

\subsection{References}

[1] Piper, G.E., Dwan, T.E., Wick, C.E., and Mitchell, E.E., "Teaching Environmental Systems Engineering," 1998 ASEE Annual Conference Proceedings, Seattle, WA, 1998.

[2] Piper, G.E., Dwan, T.E., Wick, C.E., and Mitchell, E.E., "Environmental Systems Engineering Course," Proceedings of the 1998 Summer Computer Simulation Conference, Reno, NV, 1998.

[3] MATLAB User's Guide, The Mathworks, Inc., Natick, MA 1990.

\subsection{Biographical Information}

GEORGE E. PIPER is an Associate Professor of Systems Engineering at the United States Naval Academy. He joined the Naval Academy faculty in January 1994. He holds a B.S., M.S. and a Ph.D. in mechanical engineering from Drexel University. Prior to joining the Naval Academy faculty, Dr. Piper was a senior member of the technical staff at Martin Marietta's Astro Space Division. He is a licensed Professional Engineer. Dr. Piper's current interests include space vehicle dynamics and control. He also keeps an active interest in engineering design and environmental engineering issues.

JENNIFER K. WATERS is an Assistant Professor of Ocean Engineering in the NAOME Department at USNA. She joined the USNA faculty in September 1994. She teaches primarily core and elective ocean engineering courses, and is involved in various ocean engineering and naval architecture research projects. Before joining the faculty at USNA, Dr. Waters worked as a research assistant at Davidson Laboratory, where she was involved in water quality prediction and monitoring projects, beach erosion studies, and towing tank experiments. Prior to that, while a student at Webb Institute, she spent her winters working as a ship fitter at Newport News Shipyard, as a 
cadet aboard the MormacSun, and as a Junior Naval Architect at small naval architecture design firms in the U.S. and Australia.

TERRENCE E. DWAN is a Professor of Systems Engineering at the United States Naval Academy. He has been a member of the Naval Academy faculty for fourteen years. He has a B.S. degree in Electrical Engineering from the University of Oklahoma, an M.S. in E.E. from Northeastern University and a Ph.D. from the University of Wyoming. His areas of interest are environmental systems, modeling, signal processing and control.

KIRIAKOS KIRIAKIDIS was born in Patras, Greece, in October 1967. He received the Diploma in mechanical engineering (1990) from the National Technical University of Athens, Greece, and the M.S. (1993) and Ph.D. (1996) degrees, also in mechanical engineering, from Polytechnic University, Brooklyn, NY. From 1995 to 1996, he was an Instructor in the Department of Mechanical Engineering at Polytechnic University. Since 1996, he has been an Assistant Professor of Systems Engineering at United States Naval Academy, Annapolis, MD. His research interests are in the field of fuzzy--model--based control, LMIs, and combustion systems. Dr. Kiriakidis is a member of the Technical Chamber of Greece. 